\title{
Robot Assisted Training for Upper Limbs using Impedance Control based on Iterative Learning
}

\author{
Kamran Maqsood ${ }^{1}$, Jingkang Xia ${ }^{2}$, Deqing Huang ${ }^{2}$, Yanan $\mathrm{Li}^{1}$ \\ 1. School of Engineering and Informatics, University of Sussex, Brighton, BN1 9RH, UK. \\ E-mail: y1557@ sussex.ac.uk \\ 2. School of Electrical Engineering, Southwest Jiaotong University, Chengdu 610031, P. R. China.
}

\begin{abstract}
This paper proposes an approach to improve robot assisted physical training subject to human uncertainties. This approach is based on impedance control which is used to regulate the dynamic relationship between the robot's position and contact force. Repetitive exercise is considered and impedance parameters are adapted in accordance with the human user to provide physical training as needed. Different from the existing approaches, the proposed one has the capacity to deal with time-length-varying cycles, which is a critical issue in physical training of human's upper limbs. By theoretical analysis and experimental results, we show that the approach can effectively learn the required robot's impedance parameters and improve the performance of physical training.
\end{abstract}

Key Words: Robot Assisted Training, Impedance Control, Iterative Learning

\section{INTRODUCTION}

Among the causes of disability, stroke is a leading one in the world. The most common approach to address stroke is one to one guided exercise through physiotherapy. However, this conventional approach is no more considered to be advantageous due to reasons like labour-intense and training sessions often shorter than required. In addition to this, therapy differs from one therapist to another based on the therapist's experience and expertise [1]. Therefore, in the past few decades, many researchers have studied robotic rehabilitation [2].

Robotic applications in rehabilitation therapy can provide interactive training tasks that improve therapy performance and recovery. Inclusively, the availability of intensive and repetitive physical training/therapy significantly diminishes the burden of physiotherapists [3, 4]. Proportional and derivative control is the conventional technique used for the rehabilitation devices [5]. Linear control techniques are not really the solution due to the difficulty in obtaining the required results even if these approaches are used with nonlinear disturbances accounted [6]. Computed torque controller is used to control rehabilitation robots, with degraded performance in uncertain environment dynamics. To resolve these problems, many other techniques are introduced, such as adaptive control [6], fuzzy control [7] and sliding mode control [8].

Adaptability is a major concern for both interactive parties during upper-limb rehabilitation training [9]. Therefore, an adaptable control system is a key requirement in rehabilitation robot design. Impedance control is one of the reliable and effective control technologies, which regulates interaction force through settling down the dynamic relation between patient and end-effector $[10,11,12]$. In addition to this, rehabilitation robots must be capable of guiding the patient gently towards a target, and they must show essentially adaptive impedance during task completion [13]. As compared to other control algorithms based on position, force and hybrid force/position control, impedance control has three advantages. First, its main objective is to control the dynamics between contact force and motion, instead of controlling different variables separately. Second, in constrained motion, free motion and even in transient progression, impedance control is useful without any mode switching. Lastly, impedance control integrated with iterative learning has a novel ability to provide an optimal robotic behaviour through repetitive human-robot interaction, which will be discussed in detail in the following sections.

One feature in robot rehabilitation training applications is repeatability, where a reference trajectory can repeat over a given operating time. A repetitive motion control method is proposed for robot manipulators, where the key objective is to track a desired periodic trajectory through repeated learning cycles [14]. Another research proposes a hybrid method which consists of iterative learning and model-based control to attain better quality motion control for repetitive tasks [15]. There have been research works in which iterative learning and impedance control are combined. An iterative learning method for impedance control is studied in [16]. Impedance control with neural network based learning is investigated in [17]. The convergence of the robot's dynamics to a desired impedance model is achieved through an iterative learning method [18]. Different from these works, one issue in robot assisted upper limb rehabilitation is that a human patient cannot guarantee the same time duration to complete a task through interaction with the robot, which violates the periodicity condition that is critical for many iterative learning methods. In this paper, we will resolve this problem by incorporating a recent 
research on iterative learning control (ILC) with a varying time length $[19,20]$. We will validate the impedance learning for upper limb rehabilitation even for a human patient completing each cycle with an indeterminate period. We will also demonstrate how the robot adapts to human subjects' impedance parameters with different capabilities to complete a reaching task. The mathematical derivation and proof of concept by simulations have been reported in our previous work [21] and this paper will investigate its implementation on a physical robotic platform.

The remainder of this paper is organized as follows. In Section II, the research problem of time-length-varying iterative learning is explained and the proposed approach is outlined. In Section III, the experimental setup and graphic user interface (GUI) are introduced. In section IV, various human behaviours in physical training are considered and the proposed approach is verified through experimental results. In Section V, future study and conclusions of the proposed work are discussed.

\section{Problem Formulation and Proposed Approach}

In this paper, robot assisted physical training for upperlimbs is studied. The proposed algorithm will be implemented on a robotic platform as shown in Fig. 1. A healthy human subject practices a predefined movement by holding the robot's handle, emulating a patient's various behaviours as will be discussed in the experiment section. The robot is controlled by an impedance controller to be also introduced later. While the human performance changes during the exercise in different cycles, the robot is expected to learn that change and adapt its assistance when repeating the training process with respect to different behaviours (e.g. active, passive).

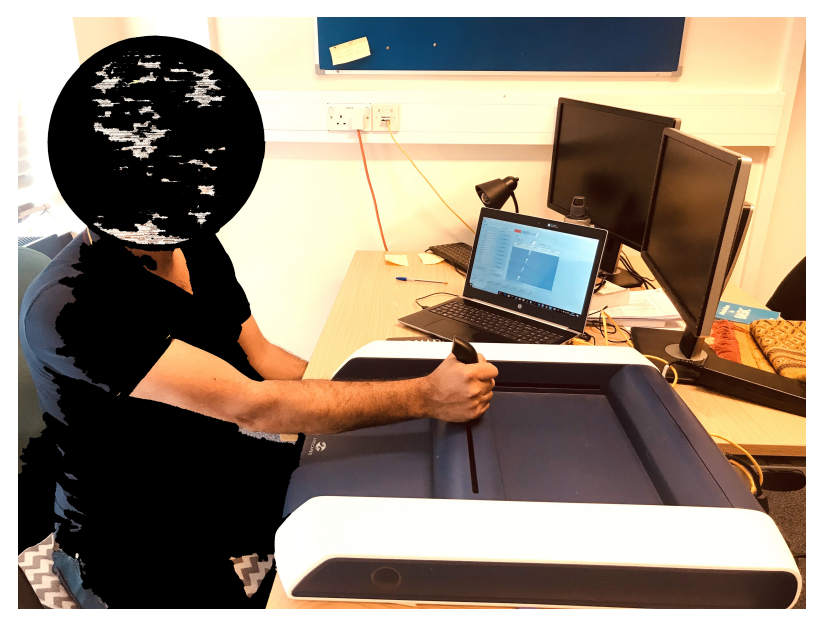

Figure 1: Robot assisted physical training scenario. A human user holds the handle mounted on a H-shaped robotic platform, while the movement of that handle is determined by the force exerted by the human hand and also the torques by internally embedded motors to reach a desired position through a specified trajectory.

In the rest of this section, we will first mathematically describe the robot dynamics. Then, the learning control problem will be formulated on the basis of the human model's unknown parameters. At last, the proposed learning approach is outlined and elaborated.

\subsection{Problem Statement}

The robot dynamics for rehabilitation can be described as

$$
M_{j}(q) \ddot{q}+C_{j}(q, \dot{q}) \dot{q}+G_{j}(q)=\tau+J^{T}(q) u_{h}
$$

where $q$ is the robot's coordinate in the joint space, $C_{j}(q, \dot{q}) \dot{q}$ is the Coriolis and centrifugal torque, $G_{j}(q)$ is the gravitational torque, $M_{j}(q)$ is the inertia matrix, $\tau$ is the joint torque supplied by the actuators, $u_{h}$ is the force applied by the human and $J(q)$ is the Jacobian matrix. ${ }^{T}$ stands for matrix transpose. In the task space, robot's dynamics can be obtained through kinematic transformation as

$$
M(q) \ddot{x}_{a}+C(q, \dot{q}) \dot{x}_{a}+G(q)=u_{r}+u_{h}
$$

where $x_{a}$ is the robot handle's actual position, and $M(q)$, $C(q, \dot{q}), G(q)$ and $u_{r}$ are respectively obtained as

$$
\begin{array}{r}
M(q)=J^{-T}(q) M_{j}(q) J^{-1}(q) \\
C(q, \dot{q})=J^{-T}(q)\left(C_{j}(q, \dot{q})\right. \\
\left.-M(q) J^{-1}(q) \dot{J}(q)\right) J^{-1}(q) \\
G(q)=J^{-T}(q) G_{j}(q), u_{r}=J^{-T}(q) \tau
\end{array}
$$

From Eq. (2), it is clearly seen that the robot's movements are determined by both human's and robot's control inputs $u_{h}$ and $u_{r}$. For a rehabilitation system where the human subject is expected to actively anticipate training tasks, the robot needs to learn about human strength and provide assistance as necessary. Therefore, designing the robot controller $u_{r}$ is directly dependent on the human's control input $u_{h}$, whose model needs to be studied.

As physical rehabilitation training typically involves a trajectory tracking task, the human's control input is constructed as follows:

$$
u_{h}=-K_{x h}\left(x_{a}-x_{t}\right)-K_{b h} \dot{x}_{a}
$$

where the human's stiffness parameters and damping parameters are $K_{x h}$ and $K_{b h}$ respectively and the desired trajectory is $x_{t}$ which is predefined for a task. The above equation explains how a human can accomplish the desired task to some extent, and human performance can be determined by the parameters $K_{b h}$ and $K_{x h}$ that are unknown to the training robot. As physical rehabilitation training is usually based on a repetitive procedure with multiple cycles, these parameters can be assumed to have the following property:

$$
K_{x h}^{j}(t)=K_{x h}^{j-1}(t), K_{b h}^{j}(t)=K_{b h}^{j-1}(t)
$$

where $j$ is the cycle number and $t \in\left[0, T_{m}\right]$ with $T_{m}$ as a time duration. For convenience, ${ }^{j}$ is not used in the remaining part of paper.

In conventional works of iterative learning control (ILC) $[22,23], T_{m}$ is assumed to be a fixed time duration. Different from those works, here we assume that each task cycle has a different time length $T^{j}$. This assumption is necessary in robot assisted rehabilitation, since a human 
patient typically cannot repeat a movement with the same time duration in every cycle. This uncertain behaviour of completing movements in different time durations makes the robot controller design challenging. In particular, if $T^{j}$ is smaller than $T_{m}$, there are no data between $T^{j}$ and $T_{m}$ that can be used in the next cycle; if $T^{j}$ is larger than $T_{m}$, how to use data after $T_{m}$ for learning control needs to be addressed. These problems will be studied in this paper so that the robot can effectively learn from the previous cycles for improvement of the human's physical exercise.

\subsection{Proposed Approach}

In this subsection, we introduce the robot's controller that assists the human partner in tracking a desired trajectory, while it evaluates the human partner's performance by iterative learning subject to the human's uncertainty. The mathematical derivation of the proposed approach with simulation results has already been discussed in [21], and the proposed approach is outlined to make this paper selfcontained.

The robot's controller is designed as

$$
u_{r}=\hat{K}_{h x} e+\hat{K}_{b h} \dot{x}_{a}
$$

where $\hat{K}_{h x}$ and $\hat{K}_{b h}$ are the robot's impedance parameters, and $e=x_{a}-x_{t}$ is the tracking error. Stiffness $\hat{K}_{h x}$ is the major parameter that is considered to improve the tracking performance in this proposed research. Damping $\hat{K}_{b h}$ is an important factor to make smooth movements during exercise.

First of all, without loss of generality, we assume $T_{m}$ is large enough so that $T^{j} \leq T_{m}$ always holds. Then, we define $\varepsilon=\dot{e}+\alpha e$ which will be used to verify the convergence of impedance parameters. Then, for $t \leq T^{j}$, the learning laws of impedance parameters are designed as

$$
\begin{aligned}
\hat{K}_{h x}^{j}(t) & =\hat{K}_{h x}^{j-1}(t)-\beta_{1} \varepsilon(t) e^{T}(t) \\
\hat{K}_{b h}^{j}(t) & =\hat{K}_{b h}^{j-1}(t)-\beta_{2} \varepsilon \dot{x_{a}}{ }^{T}(t)
\end{aligned}
$$

These learning laws are developed using iterative learning, in which impedance parameters are updated based on their values in the previous cycle. Position and velocity errors ( $e$, $\dot{e})$ are used in the learning so that optimal values of stiffness $K_{h x}$ and damping $K_{b h}$ will be obtained when the tracking task is achieved. $\beta_{1}$ and $\beta_{2}$ are two constant learning rates. In this case of $t \leq T^{j}$, the proposed learning laws work with the movement uncertainty, on the basis of when the human finishes each exercise cycle. In particular, the update of impedance will be terminated with the completion of the human's movement.

For $T^{j}<t \leq T_{m}$, the learning laws are designed as

$$
\hat{K}_{h x}^{j}(t)=\hat{K}_{h x}^{j-1}(t), \hat{K}_{b h}^{j}(t)=\hat{K}_{b h}^{j-1}(t)
$$

which indicate that the impedance parameters keep the same after $T^{j}$. Because there are no data between $T^{j}$ and $T_{m}$ that can be used in the next cycle, $\beta_{1}$ and $\beta_{2}$ in Eq. (7) are set as zero for this case, leading to the learning laws in Eq. (8).

The controller design for this approach is shown using the block diagram in Fig. 2. The robot's control input is $u_{r}$ mentioned above in Eq. (6). Its impedance parameters are updated based on iterative learning for two cases due to varying-length time durations. The learning laws are based on position and velocity errors, indicating that the learning convergence will be obtained when the tracking task is achieved.

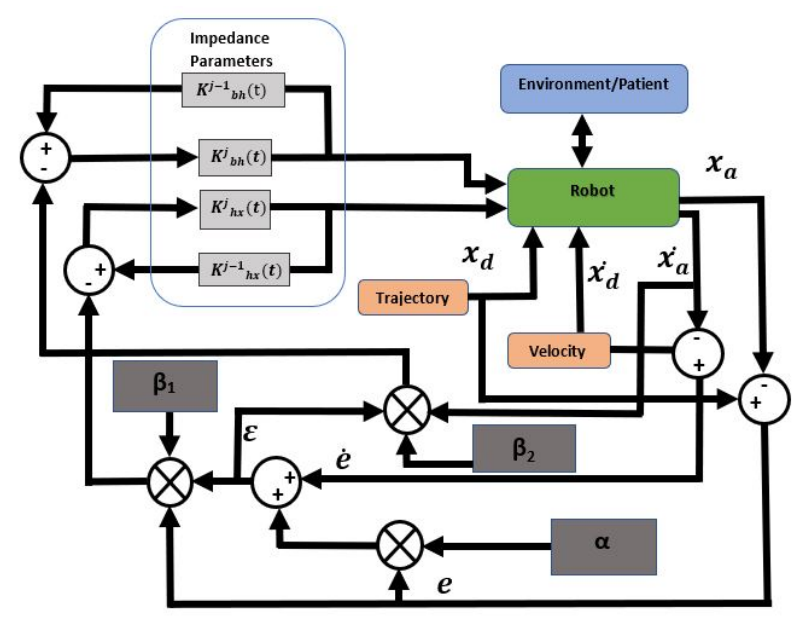

Figure 2: The proposed controller.

\section{Experimental Setup}

To verify the profits of proposed controller, it is implemented on a 2 degrees of freedom (DOF) rehabilitation robot H-MAN as shown in Fig. 1. Primary advantages of this robotic platform include its lightweight mechanics, simple characteristics and basic shaped workspace that can easily be used for different applications [24]. Movement of the mounted handle is achieved by a simple kinematic topology in which two actuators drive respecting perpendicular linear sliders. The torque from actuators is transmitted to the handle through a $\mathrm{H}$-shaped cable driven transmission system.

Interfacing of H-MAN is done by using $\mathrm{C}$ sharp windows application, which is connected to the hardware through RJ45 connection. Coordinate settings and initial position calibration can be specified by this interface, while the proposed algorithm will automatically update the impedance parameters according to the training human and required rehabilitation exercise. Data such as position and velocity of the handle can be stored to evaluate the performance of the proposed controller, as shown in the next section.

The robot's reference trajectory for the exercises is given by

$$
x_{d}=A \sin (\omega t)
$$

where $A=150 \mathrm{~mm}, \omega=\pi / 3 \mathrm{rad} / \mathrm{s}$ and $t \in[0,3] \mathrm{s}$. This desired trajectory represents a complete cycle including forward and backward movements of the handle in $x$ axis. The initial stiffness and damping parameters in the first cycle are $\hat{K}_{h x}=200 \mathrm{~N} / \mathrm{m}, \hat{K}_{b h}=50 \mathrm{Nm} / \mathrm{s}$, respectively. These values are set to ensure moderate tracking performance with smooth movements, as will be shown in the results section. The stiffness parameter is updated on the basis of previous cycle value, but the damping parameter 
is deliberately kept constant to ensure a smooth movement. Therefore, the robot's control parameters are $\Gamma=0.5$, $\alpha=100$ and in Eq. (7) $\beta_{1}=800, \beta_{2}=0$.

\section{Results and Discussion}

\subsection{Experimental Results}

This paper evaluates a new algorithm to provide rehabilitation training to patients with different conditions. When a patient and a physiotherapist are in physical contact with each other, the physiotherapist determines the required force or assistance according to the patient's performance. The same principle is used in this proposed research, with training automatically adapted to different patient conditions.

\subsubsection{No Interaction}

In this condition, without human user the robot moves to track a desired trajectory and attain the final target. This is a condition that is used to check the difference from other interaction types and validate the learning process. Results in this condition are shown in Figs. 3, 4 and 5 which illustrate that the actual trajectory can track the desired one with errors decreasing over cycles, and the robot's stiffness shows convergence after a few cycles. In Fig. 5, a drop is found just before 2 seconds, which is when the first target is reached so after that the stiffness increases till the achievement of the second target.

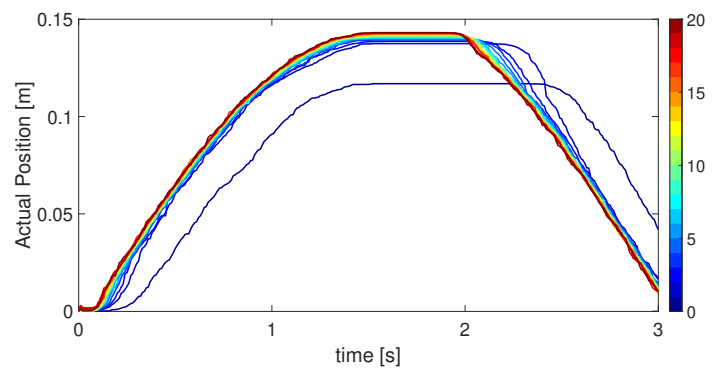

Figure 3: Desired trajectory followed by the robot without interaction. Improvement of the actual trajectory is shown by the color bar, which changes from blue (cycle number $j=1)$ to $\operatorname{red}(j=20)$.

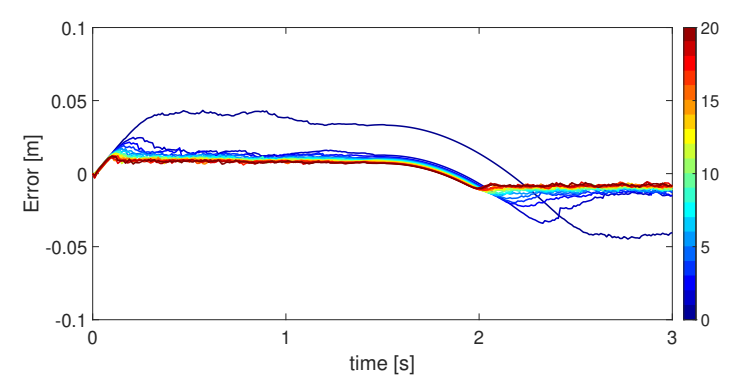

Figure 4: Tracking error between desired and actual trajectories in the condition of no interaction. The reduction in error is shown by the color bar, which changes from blue (cycle number $j=1)$ to red $(j=20)$.

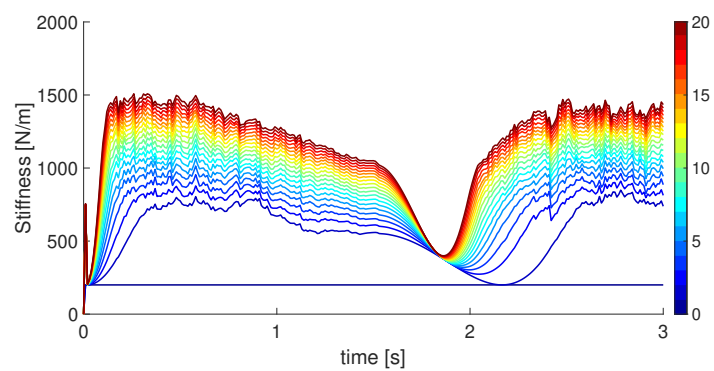

Figure 5: Stiffness during target reaching task in the condition of no interaction. The learning process is shown by the color bar, which changes from blue (cycle number $j=1$ ) to $\operatorname{red}(j=20)$.

\subsubsection{Passive Interaction}

This condition requires the therapist or robotic device to perform exercise without any contribution from the patient, i.e. the patient's hand is physically connected to the robot handle and does not intentionally apply any force. In Figs. 6 and 7, it is found that actual trajectory and error show the same trend as in the no-interaction condition. However, Fig. 8 shows stiffness increase because of affecting mass of the human arm. In other words, the robot needs more stiffness to track the trajectory as compared to the no-interaction condition.

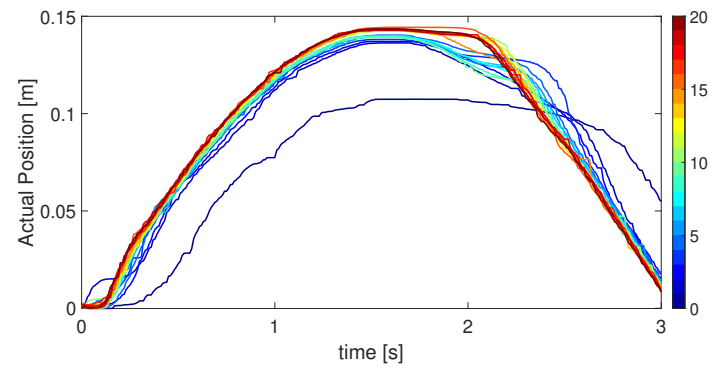

Figure 6: Desired trajectory followed by the robot with passive interaction. Improvement of the actual trajectory is shown by the color bar, which changes from blue (cycle number $j=1)$ to red $(j=20)$.

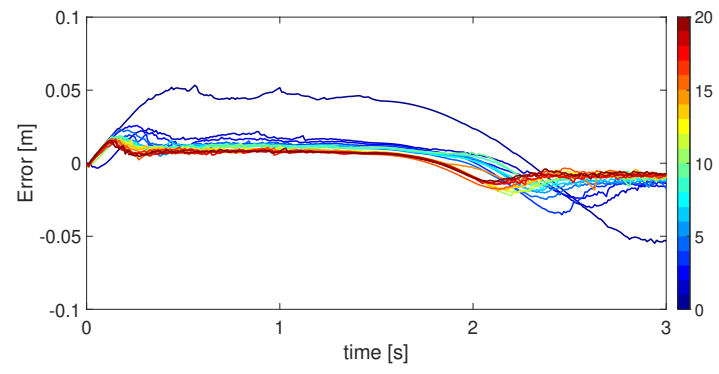

Figure 7: Tracking error between desired and actual trajectories in the condition of passive interaction. The reduction in error is shown by the color bar, which changes from blue (cycle number $j=1)$ to red $(j=20)$. 


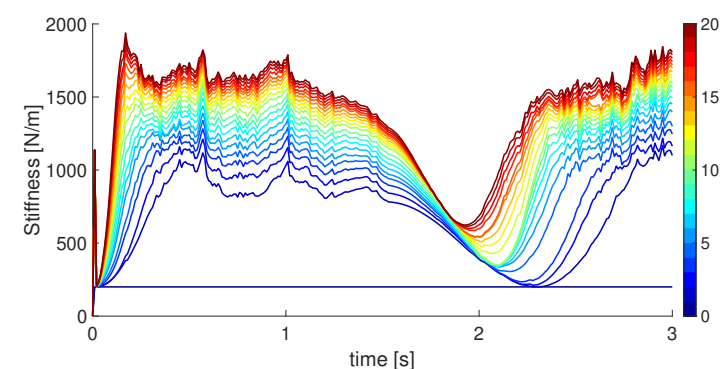

Figure 8: Stiffness during target reaching task in the condition of passive interaction. The learning process is shown by the color bar, which changes from blue (cycle number $j=1)$ to red $(j=20)$.

\subsubsection{Active Interaction (Assistance)}

Active interaction indicates a condition where the patien$\mathrm{t}$ has little strength but still requires assistance. The robot should provide a minimum possible force to produce the specified motion as not to assist more than needed. The algorithm needs to deal with varying time lengths as the human patient introduces the uncertainty. With the proposed learning algorithm, if the patient completes his exercise cycle in a shorter time, impedance control parameters will be changed with the new updated values till $t$. In the next cycle, the algorithm uses the learned values till time $t$ and the old values after $t$ will be maintained for the remaining cycle. The results in Figs. 10 and 11 show that every cycle is completed with a different time duration. In addition to this, as compared to conditions of passive interaction and no interaction, the robot's stiffness converges to a smaller value showing less assistance to the human user so it encourages the human user to contribute more.

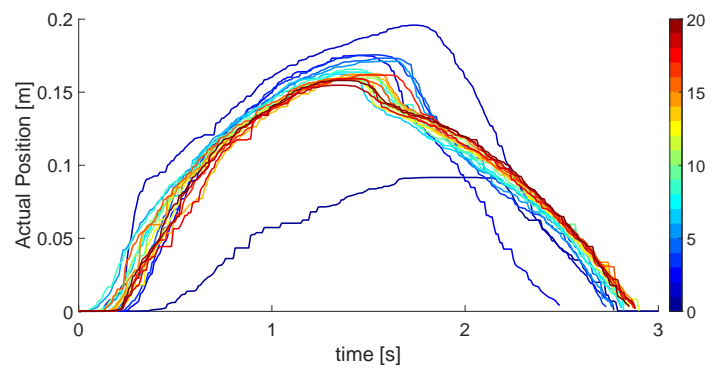

Figure 9: Desired trajectory followed by the robot with active interaction. Improvement of the actual trajectory is shown by the color bar, which changes from blue (cycle number $j=1)$ to red $(j=20)$.

\subsubsection{Active Interaction (Less Assistance)}

This condition tests a case when the patient has more active motor function, so the robot should provide less assistance to improve the patient's training performance. In this condition, usually the patient can complete a training cycle in a shorter time as compared to the above conditions. Therefore, this uncertainty needs to be handled by the proposed learning method. The robot should provide assistance as

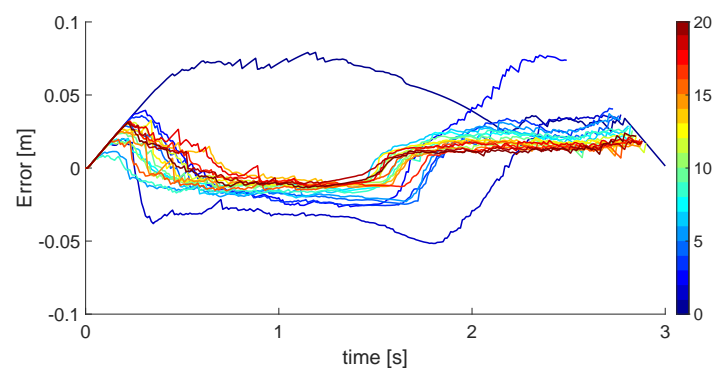

Figure 10: Tracking error between desired and actual trajectories in the condition of active interaction. The reduction in error is shown by the color bar, which changes from blue (cycle number $j=1)$ to red $(j=20)$.

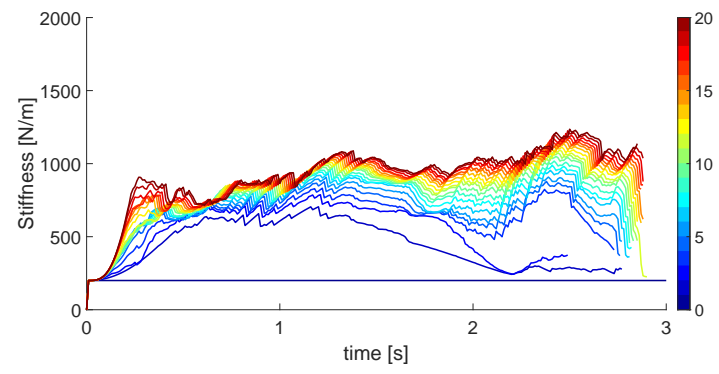

Figure 11: Stiffness during target reaching task in the condition of active interaction. The learning process is shown by the color bar, which changes from blue (cycle number $j=1)$ to red $(j=20)$.

little as possible to achieve a specified motion by the patient him-/herself. Results in Figs. 12 and 13 show that the robot allows the human user to complete an cycle earlier than predefined. In particular, if the human user completes a cycle in time then impedance parameters are recorded for the next cycle but do not change for the remaining of the current cycle. However, in the next cycle the recorded impedance parameters may be required if the human user takes more time to complete the cycle. As compared to passive interaction and active interaction (more assistance), this condition achieves further decreasing stiffness indicating less assistance to the human user, as shown in Fig. 14.

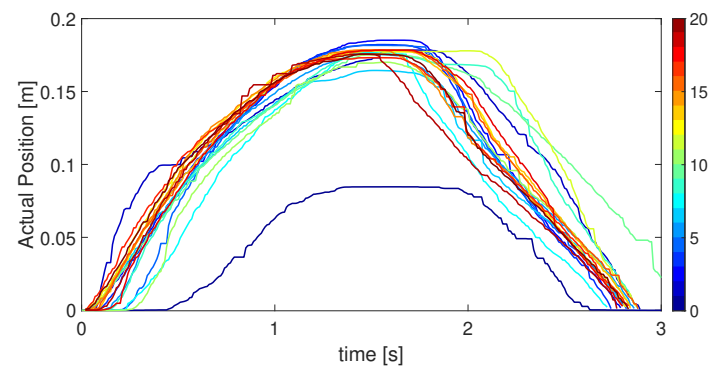

Figure 12: Desired trajectory followed by the robot with active interaction (less assistance). Improvement of the actual trajectory is shown by the color bar, which changes from blue (cycle number $j=1)$ to red $(j=20)$. 


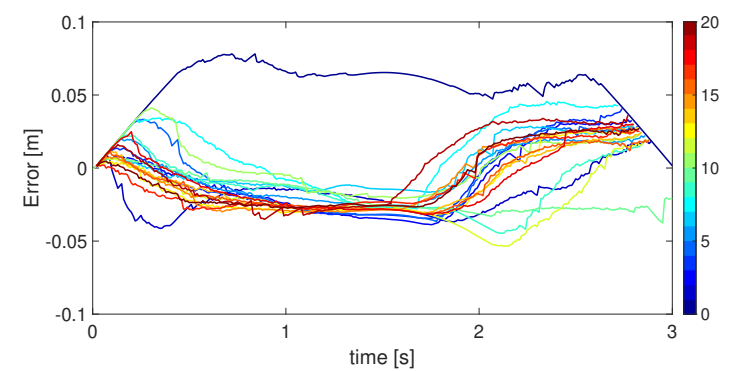

Figure 13: Tracking error between desired and actual trajectories in the condition of active interaction (less assistance). The reduction in error is shown by the color bar, which changes from blue (cycle number $j=1$ ) to red $(j=20)$.

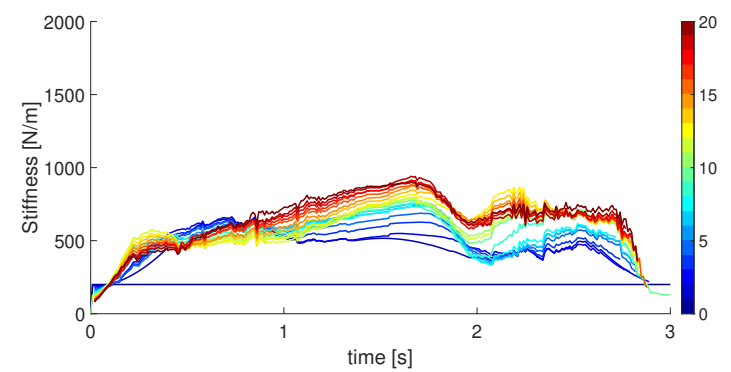

Figure 14: Stiffness during target reaching task in the condition of active interaction (less assistance). The learning process is shown by the color bar, which changes from blue (cycle number $j=1)$ to red $(j=20)$.

\subsection{Convergence of Stiffness}

In this experiment, we test the convergence of stiffness with a different initial value $1200 \mathrm{~N} / \mathrm{m}$ in the same condition of active interaction (less assistance). Results in Figs. 15 and 16 show that the stiffness decreases to a level similar to that in the previous experiment with an initial value $200 \mathrm{~N} / \mathrm{m}$ smaller than the converging one. These results demonstrate that the proposed learning method can automatically find an optimal impedance that helps the human user's training, which converges to the same level regardless of its initial value.

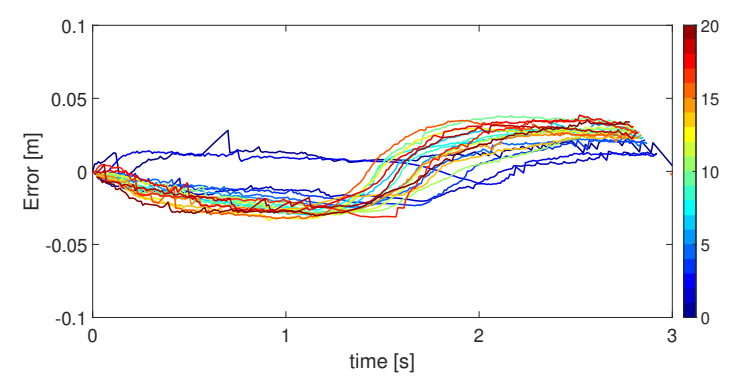

Figure 15: Tracking error between desired and actual trajectories in the condition of active interaction (less assistance) with initial stiffness of 1200 . The reduction in error is shown by the color bar, which changes from blue (cycle number $j=1)$ to red $(j=20)$.

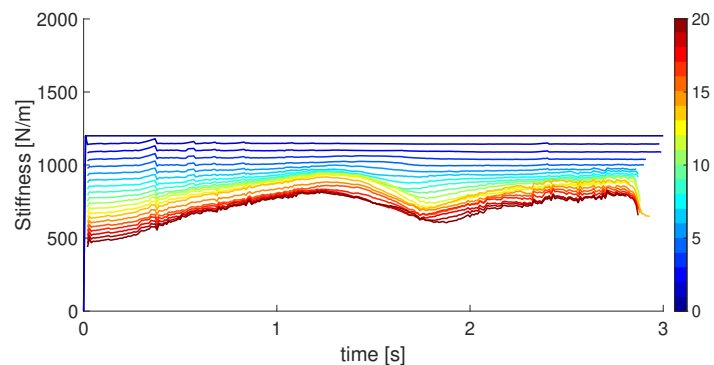

Figure 16: Stiffness during target reaching task in the condition of active interaction (less assistance) with initial stiffness of 1200. The learning process is shown by the color bar, which changes from blue (cycle number $j=1$ ) to $\operatorname{red}(j=20)$.

\section{Conclusion}

In this proposed research, impedance learning for rehabilitation training has been studied. Considering the complexity of human dynamics and repetitive natures of rehabilitation, model-free iterative learning control (ILC) is adopted to develop an impedance learning algorithm. A novel issue of human variance in repetitive movements during physical exercises is addressed by using varying length ILC. Preliminary experimental results show the validity of the proposed method in terms of dealing with uncertain cycle lengths, adapting to different human behaviours and ensuring learning convergence. One of our future works will be implementation of this method for upper-limb rehabilitation of real patients, and evaluation of its capability in assisting the patients' recovery.

\section{REFERENCES}

[1] N. Schweighofer, C. Wang, D. Mottet, I. Laffont, K. Bakthi, D. J. Reinkensmeyer, and O. Rémy-Néris, "Dissociating motor learning from recovery in exoskeleton training post-stroke," Journal of neuroengineering and rehabilitation, vol. 15, no. 1, p. 89, 2018.

[2] S. Haddadin and E. Croft, "Physical human-robot interaction," in Springer handbook of robotics, pp. 1835-1874, Springer, 2016.

[3] R. Riener, T. Nef, and G. Colombo, "Robot-aided neurorehabilitation of the upper extremities," Medical and biological engineering and computing, vol. 43 , no. 1, pp. 2-10, 2005.

[4] R. Colombo, I. Sterpi, A. Mazzone, C. Delconte, and F. Pisano, "Improving proprioceptive deficits after stroke through robot-assisted training of the upper limb: a pilot case report study," Neurocase, vol. 22, no. 2, pp. 191-200, 2016.

[5] M. Babaiasl, S. H. Mahdioun, P. Jaryani, and M. Yazdani, "A review of technological and clinical aspects of robotaided rehabilitation of upper-extremity after stroke," Disability and Rehabilitation: Assistive Technology, vol. 11, no. 4, pp. 263-280, 2016.

[6] J. Hill and F. Fahimi, "Active disturbance rejection for walking bipedal robots using the acceleration of the upper limbs," Robotica, vol. 33, no. 2, pp. 264-281, 2015.

[7] X. Jiang, Z. Wang, C. Zhang, and L. Yang, "Fuzzy neural network control of the rehabilitation robotic arm driven 
by pneumatic muscles," Industrial Robot: An International Journal, vol. 42, no. 1, pp. 36-43, 2015.

[8] M. H. Rahman, M. Saad, J.-P. Kenné, and P. S. Archambault, "Control of an exoskeleton robot arm with sliding mode exponential reaching law," International Journal of Control, Automation and Systems, vol. 11, no. 1, pp. 92104, 2013.

[9] M. M. Marinho, A. Nakazawa, J. Nakanishi, T. Ueyama, Y. Hasegawa, J. Arata, M. Mitsuishi, and K. Harada, "Conceptual design of a versatile robot for minimally invasive transnasal microsurgery," in 2016 International Symposium on Micro-NanoMechatronics and Human Science (MHS), pp. 1-3, IEEE, 2016.

[10] T. Wang and A. Song, "An adaptive iterative learning based impedance control for robot-aided upper-limb passive rehabilitation," Frontiers in Robotics and AI, vol. 6, p. 41, 2019.

[11] K. Kiguchi, K. Iwami, M. Yasuda, K. Watanabe, and T. Fukuda, "An exoskeletal robot for human shoulder joint motion assist," IEEE/ASME transactions on mechatronics, vol. 8, no. 1, pp. 125-135, 2003.

[12] W. Meng, Q. Liu, Z. Zhou, Q. Ai, B. Sheng, and S. S. Xie, "Recent development of mechanisms and control strategies for robot-assisted lower limb rehabilitation," Mechatronics, vol. 31, pp. 132-145, 2015.

[13] H. I. Krebs, J. J. Palazzolo, L. Dipietro, M. Ferraro, J. Krol, K. Rannekleiv, B. T. Volpe, and N. Hogan, "Rehabilitation robotics: Performance-based progressive robot-assisted therapy," Autonomous robots, vol. 15, no. 1, pp. 7-20, 2003.

[14] K. Kaneko and R. Horowitz, "Repetitive and adaptive control of robot manipulators with velocity estimation," IEEE Transactions on Robotics and Automation, vol. 13, no. 2, pp. 204-217, 1997.

[15] B. Bukkems, D. Kostic, B. De Jager, and M. Steinbuch, "Learning-based identification and iterative learning control of direct-drive robots," IEEE Transactions on Control Systems Technology, vol. 13, no. 4, pp. 537-549, 2005.

[16] P. T. A. Nguyen, H.-Y. Han, S. Arimoto, and S. Kawamura, "Iterative learning of impedance control," in Proceedings 1999 IEEE/RSJ International Conference on Intelligent Robots and Systems. Human and Environment Friendly Robots with High Intelligence and Emotional Quotients (Cat. No. 99CH36289), vol. 2, pp. 653-658, IEEE, 1999.

[17] T. Tsuji, K. Ito, and P. G. Morasso, "Neural network learning of robot arm impedance in operational space," IEEE Transactions on Systems, Man, and Cybernetics, Part B (Cybernetics), vol. 26, no. 2, pp. 290-298, 1996.

[18] Y. Li, S. Ge, and C. Yang, "Learning impedance control for physical robot-environment interaction," International Journal of Control, vol. 85, no. 2, pp. 182-193, 2012.

[19] D. Shen and J.-X. Xu, "Adaptive learning control for nonlinear systems with randomly varying iteration lengths," IEEE transactions on neural networks and learning system$s$, vol. 30, no. 4, pp. 1119-1132, 2018.

[20] X. Li, J.-X. Xu, and D. Huang, "Iterative learning control for nonlinear dynamic systems with randomly varying trial lengths," International Journal of Adaptive Control and Signal Processing, vol. 29, no. 11, pp. 1341-1353, 2015.

[21] Y. Li, X. Zhou, J. Zhong, and X. Li, "Robotic impedance learning for robot-assisted physical training," Frontiers in Robotics and AI, vol. 6, p. 78, 2019.

[22] Y. Yokokohji and T. Yoshikawa, "Bilateral control of master-slave manipulators for ideal kinesthetic couplingformulation and experiment," IEEE transactions on robotics and automation, vol. 10, no. 5, pp. 605-620, 1994.
[23] Y. Li, G. Ganesh, N. Jarrassé, S. Haddadin, A. AlbuSchaeffer, and E. Burdet, "Force, impedance, and trajectory learning for contact tooling and haptic identification," IEEE Transactions on Robotics, vol. 34, no. 5, pp. 1170-1182, 2018.

[24] D. Campolo, P. Tommasino, K. Gamage, J. Klein, C. M. Hughes, and L. Masia, "H-man: A planar, h-shape cabled differential robotic manipulandum for experiments on human motor control," Journal of neuroscience methods, vol. 235, pp. 285-297, 2014. 